\title{
Configuration space in electron glasses
}

\author{
A. Pérez-Garrido, M. Ortuño, A. M. Somoza and A. Díaz-Sánchez \\ Departamento de Física, Universidad de Murcia, Murcia 30.071, Spain
}

\begin{abstract}
We study numerically the configuration space at low energy of electron glasses. We consider systems with Coulomb interactions, short-range interactions and no interactions. First, we calculate the integrated density of configurations as a function of energy. At a given energy, this density is smaller for Coulomb glasses than for short-range systems, which in turn is smaller than for noninteracting systems. We analyze how the site occupancy varies with the number of configurations. Through this study we estimate the number of particles involved in a typical low-energy transition between configurations. This number increases with system size for long range interactions, while it is basically constant for a short-range interaction. Finally we calculate the density of metastable configurations, i.e. valleys, classified according to their degree of stability.
\end{abstract}

PACS numbers: 71.10-w, 73.61.Jc

\section{INTRODUCTION}

The term Coulomb glass refers to Anderson insulators with Coulomb interactions between the localized electrons, and it has established itself as an important model in the study of the electronics properties of insulators [1]. It assumes that quantum energies $t$ arising from tunneling are much smaller than the other important energies in the problem, i.e. Coulomb interactions and random energy fluctuations. In this situation the Hubbard energy is very large and practically forbids double occupation of sites. The interesting case then corresponds to a number of electrons roughly half the number of sites.

Until a few years ago, most studies of the Coulomb glass consisted mainly of finding the ground state of the system, and considering from there on only one-particle transitions [2-4]. The main problem arising with this is the neglection of many-body effects. Several experimental [5-7] and numerical [8] results give evidences that electronic correlations are very important in these systems and they must be taken into account. More recently, methods were developed to obtain an almost complete set of low-lying states of the Coulomb glass $[9,10]$. This process allows for a much more detailed consideration of many-body effects.

A proper understanding of the low-energy configuration space is extremely important for the study of most physical properties. Previous works on Coulomb glasses have never focus on the properties and relations between configurations. We only have an indirect knowledge of them through the analysis of different physical problems. In this work we perform a systematic study of the low-energy configurations of three-dimensional Coulomb glasses. For the sake of comparison, we have also computed the same properties for non-interacting systems and for disordered systems with short range interactions.

In the next section, we introduce the model used and details of the numerical simulations. In section III, we study the entropy of the systems and compare to that obtained by two different analytical models. In section IV, we obtain the average difference in occupancy between the ground state and configurations as a function of energy, and in section $\mathrm{V}$ we calculate the average number of electrons participating in a typical low-energy transition. In section VI we study the density of valleys in configuration space. Finally, in section VII we extract some conclusions.

\section{NUMERICAL MODEL}

We consider three-dimensional systems deep in the insulating regime, where the relevant energies in the problem are the Coulomb interaction and the disorder energy. This corresponds to the condition $a \ll r_{0}$, where $a$ is the localization radius, $r_{0}=(4 \pi n / 3)^{-1 / 3}$ is the typical distance between sites and $n$ the concentration of sites.

We use the standard tight-binding Hamiltonian:

$$
H=\sum_{i} \epsilon_{i}\left(n_{i}-K\right)+\sum_{i<j} V_{i j}
$$

$\epsilon_{i}$ are the site random energies, uniformly distributed at random in the interval $(-W / 2, W / 2), W$ being the disorder strength. We take the number of electrons to be half the number of sites and $K=0.5$. The sites are arranged at random, but with a minimum separation between them, which we choose to be $0.5 r_{0}$. We take $e^{2} / r_{0}$ as our unit of energy and $r_{0}$ as our unit of distance. $V_{i j}$ is the interaction potential. In this work we use three types of potentials, coulombic, short range and non-interacting. The Coulomb potential is given by the expression

$$
V_{i j}=\frac{\left(n_{i}-K\right)\left(n_{j}-K\right)}{r_{i j}},
$$

where $r_{i j}$ is the separation between sites $i$ and $j$. For the short range potential we choose

$$
V_{i j}=\left(n_{i}-K\right)\left(n_{j}-K\right)\left(\frac{\sigma}{r_{i j}}\right)^{4}
$$


$\sigma$ was taken equal to 0.7 . Finally, the non-interacting case simply corresponds to $V_{i j}=0$.

A numerical algorithm developed to obtain the ground state and the lowest energy many-particle configurations of these systems has been used for the calculation of low energy states [11]. This algorithm considers simultaneous many-electron transitions constructed from (of the order of ten) independent one-electron transitions and perform the combination that relaxes more energy. Starting from a random initial configuration, we repeat the relaxation procedure until we cannot reduce the energy any more. The whole scheme is repeated for different initial random configurations of the charges until the configuration of lowest energy is found ten times. The configurations thus generated were memorized in terms of site occupation numbers ( 0 or 1 ) and of energy, whenever this was less than the highest energy configuration in memory storage. We complete the set of low-energy configurations by generating all the states that differ by one- or two-electron transitions from any configuration stored.

\section{NUMBER OF CONFIGURATIONS}

The first magnitude to study is the number of configurations as a function of energy. Let us call density of states to the number of one-particle states per unit energy, and density of configurations $n(E)$ to the number of many-particle configurations per unit energy. In particular, we consider the integrated density of configurations $N(E)$, i.e. the number of configurations with energy smaller than or equal to $E$. If the occupation probability of all configurations up to an energy $E$ is the same, the entropy, $S$, is then equal to $k_{\mathrm{B}} \ln N(E)$, where $k_{\mathrm{B}}$ is the Boltzmann constant which we take equal to 1 . The number of configurations strongly depends on the size of the sample, but this dependence can be roughly taken into account by an adequate normalization of the energy. The idea is to measure the energy in terms of the average energy spacing between single particle states near the Fermi level (in the absence of interactions):

$$
E=\mathcal{E} V / W
$$

where $E$ is the normalized energy, $V$ the volume of the system, $W$ the disorder strength and $\mathcal{E}$ the energy to be normalized. This procedure provides a density of configurations independent of dimensionality and system size for non interacting systems.

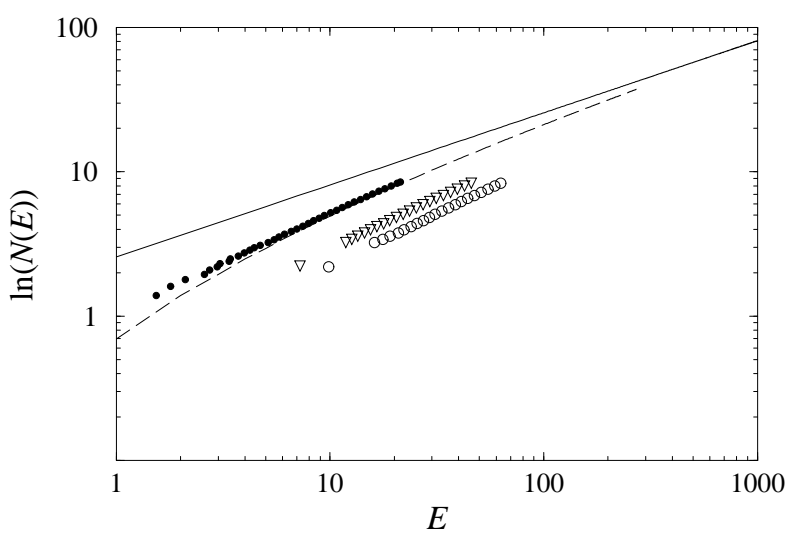

FIG. 1. $\ln N(E)$ versus energy for systems with no interactions (solid circles), short range interactions (triangles) and Coulomb interactions (empty circles). The solid line corresponds to the standard result, Eq. (8), and the dashed line to our discrete model, Eq. (15).

In Fig. 1 we plot on a double logarithmic scale the average of the entropy as a function of the normalized energy, i.e. measured in terms of the one-particle energy spacing. Averages are taken over twenty samples. The solid dots correspond to the non-interacting case, the triangles to a system with short-range interactions, and the empty dots to a Coulomb glass. As we have previously mentioned, the results for the non-interacting case are exactly independent of the sample size. The two interacting cases correspond to averages over samples with a number of sites equal to $N_{\mathrm{s}}=900$. Although, in principle, the results for interacting systems depend on size, the variation is very small and we have preferred not to show them for several sizes for the sake of clarity. In all cases considered, the fluctuations from sample to sample are large, even in the logarithmic scale considered, but averages are relatively well behaved.

For non-interacting systems it is possible to make theoretical calculations for the entropy. The classical result obtained from statistical mechanics for an infinite system with a constant density of states corresponds to the solid line in Fig. 1. The entropy is proportional to $\sqrt{E}$, and so this is a straight line with slope equal to 0.5. Although the previous result is well known, we deduce it below in order to express it in our notation and because the method of calculation can be extended to other non-standard magnitudes as we will see later on. The dashed line connects the points obtained from a finite discrete model that we also describe in the next subsection. All systems have a integrated density of the form $N(E) \propto \exp \left(E / E_{0}\right)^{\alpha}$. In all cases, for the sizes considered we checked that $\alpha \approx 0.7$. The value of the exponent, $\alpha$, is deeply affected by finite size effect. For interacting systems we are not sure of the value the exponent in the thermodynamic limit. The similarity between the interacting and non-interacting systems for the energy ranges 
considered suggests that $\alpha=0.5$ might also correspond to the thermodynamic limit for the interacting cases.

\section{A. Theoretical models}

We want to derive the entropy for a system of non interacting electrons with a constant density of states. It is possible to obtain analytically the entropy and other properties of this system for two models. Firstly, we study a continuous model using one-particle theory. Secondly, we investigate a discrete model of equally spaced levels by counting directly the number of configurations.

We first assume a continuous model with a constant density of states for the whole range of energies of interest, $g(\epsilon)=g_{0}$. The chemical potential $\mu$ and the Fermi level $\epsilon_{f}$ are equal to zero by symmetry. At a given temperature $T$, the average occupation number of a state with energy $\epsilon$ is:

$$
n_{\epsilon}=\frac{1}{1+\exp (\epsilon / T)}
$$

So the mean energy $\langle E\rangle$ of the system, measured from the ground state energy, is

$$
\langle E\rangle=\int_{-\infty}^{\infty}\left(n_{\epsilon}-\theta(\epsilon)\right) V g_{0} \epsilon d \epsilon=\frac{\pi^{2} V g_{0} T^{2}}{6},
$$

where $\theta(\epsilon)$ is the step function, which takes into account the ground state energy, and $V$ is the volume of the system. Eq. (6) allows us to change between temperature and mean energy.

The entropy $S$ of a non-interacting system can be calculated from the average site occupation through the expression [12]

$$
S=\int_{-\infty}^{\infty} V g_{0}\left\{n_{\epsilon} \ln \left(\frac{1}{n_{\epsilon}}-1\right)-\ln \left(1-n_{\epsilon}\right)\right\} d \epsilon .
$$

Integrating this expression and using Eq. (6), one obtains the entropy as a function of energy:

$$
S=\sqrt{\frac{2 \pi^{2} V g_{0}\langle E\rangle}{3}} .
$$

Eq. (8) is plotted as the solid line of Fig. 1. We can see that the numerical results do not match the theoretical one for the continuous case, Eq. (8). To check the importance of the discrete nature of our numerical systems we develop a discrete model. We now describe this discrete model for the number of configurations of non-interacting electrons with equally spaced one-particle energy levels. The dashed line in Fig. 1 represents $\log N(E)$ for this model.

Each one-particle state is labeled according to its position in the energy scale. The highest occupied level in the ground state is assigned to the zero of our scale. In the ground state, occupied levels correspond to negative numbers, while empty levels to positive numbers. A state $k$ has an energy equal to $k \Delta \epsilon$, where $\Delta \epsilon$ is the constant energy spacing between levels, which we take equal to 1 . The density of configurations $n(E)$ can be decomposed as

$$
n(E)=\sum_{i=1}^{\infty} n_{i}(E)
$$

where $n_{i}(E)$ is the number of configurations with $i$ excitations ( $i$ electrons above Fermi level and $i$ holes below it) and with a total excitation energy equal to $E$. The excitation energy of a transition from level $-m$ (with $m \geq 0)$, to level $l(l>0)$, is equal to $l-m$, recall that $\Delta \epsilon=1$. For one electron jumps, $n_{1}(k)$ is simply

$$
n_{1}(k)=k,
$$

since we have $k$ different possibles jumps of length $k$.

For $i=2$ (two excitations) the possibles jumps can be from $\epsilon_{-n}$ to $\epsilon_{j}$ and from $\epsilon_{-p}$ to $\epsilon_{l}$, with the conditions $n \neq p, j \neq l$ (from two different states to two other different states), $n, p \geq 0$ and $j, l>0$. The total energy is the sum of individual energy

$$
k=(j-n)+(l-p) .
$$

We rewrite equation (11) as

$$
k=j+l-(n+p) .
$$

$n_{2}(k)$ is, then, the number of possible combinations of $j, l, n$ and $p$ verifying expression (12). Using the function $Q_{i}$ explained below and (12) we arrive at

$$
n_{2}(k)=\sum_{j=3}^{k-1} Q_{2}(j) Q_{2}(k-j+2) .
$$

For an arbitrary $i$, the number $n_{i}(k)$ of $i$ transitions with a total energy $k$ becomes

$$
n_{i}(k)=\sum_{j=i(i+1) / 2}^{k-i(i-1) / 2} Q_{i}(j) Q_{i}(k-j+i) .
$$

The integrated density of configurations $N(k)$ is

$$
N(k)=1+\sum_{i=1}^{k} n(i)
$$

where $n(i)$ is given by Eq. (9) and where we have added 1 to count the ground state.

We have to obtain the number $Q_{i}(m)$ of possible combinations of $i$ integers whose sum is equal to $m$, and subject to the constraint that they must all be different from each other and from zero. When $i=1$ we have the trivial case 


$$
Q_{1}(m)=1,
$$

independently of $m$. It is easy to check that for $i=2$ that function becomes

$$
Q_{2}(m)=\operatorname{Int}\left(\frac{m-1}{2}\right),
$$

where $\operatorname{Int}(m)$ truncates $m$ to its integer part. When $i>2$, the function $Q_{i}(m)$ is defined in a recursive form as

$$
Q_{i}(m)=\sum_{j=1}^{\operatorname{Int}(m / i)-1} Q_{i-1}(m-i j) .
$$

A line connecting the points given by the integrated density of configurations $N(E)$ of our discrete model, obtained from Eqs. (15), (9) and (14) (where $E \equiv k \cdot \Delta \epsilon$ ), has been plotted in Fig. 1 (dashed line) as a function of energy. We can see that our predictions with the discrete model fit very well our numerical results for systems with no interactions (solid dots). Furthermore, the number of configurations of the discrete model tends to the number of configurations of the continuous model, Eq. (8), represented by the solid line in Fig. 1 .

\section{DIFFERENCE IN OCCUPATION}

An interesting quantity is the number of electrons that have to jump from the ground state to reach a given configuration, i.e., half the Hamming distance between this configuration and the ground state. We consider the average $\langle i\rangle$ of this quantity over small energy intervals and disorder realizations. For a non-interacting system this magnitude corresponds to the average occupation of states above the Fermi level, which for a (continuous) constant density of states is given by:

$$
\langle i\rangle=\int_{0}^{\infty} \frac{g_{0}}{1+\exp (\epsilon / T)} d \epsilon=g_{0} T \ln 2 .
$$

We use Eq. (6) to rewrite $\langle i\rangle$ as a function of the mean energy, instead of temperature $T,\langle E\rangle$,

$$
\langle i\rangle=\frac{\ln 2}{\pi} \sqrt{6\langle E\rangle g_{0}} .
$$

We can also calculate this quantity using our discrete model, explained before, and we get

$$
\langle i\rangle=N^{-1}(E)\left(\sum_{i=1}^{k} \sum_{j=1}^{\infty} j n_{j}(i)\right),
$$

where $N(E)$ is given by Eq. (15) and $n_{i}(E)$ by Eq. (14). In Fig. 2 we plot $\langle i\rangle$ given by Eq. (20) (solid line) and Eq. (21) (dashed line) as a function of energy. In the same figure we also represent the results obtained by numerical calculations, for a non interacting system (solid dots) and for coulombic systems (triangles correspond to 465 sites and empty squares to 899 sites). The results for non-interacting systems agree fairly well with the discrete model and, using the normalized energy, are independent of size. On the other hand, the mean number of electrons above the Fermi level is larger for systems with interactions due to the electronic correlations, confirming our results of the previous section. It is interesting to note that the results for the interacting case are also independent of the size of the system when plotted, as in Fig. 2, using the normalized energy $E$, Eq. (4) at least for the sizes considered. Both curves for Coulomb interacting systems fit to an expression of the form

$$
\langle i\rangle=C E^{\alpha},
$$

with an exponent $\alpha$ roughly equal to 0.25 , half the value for non-interacting systems.

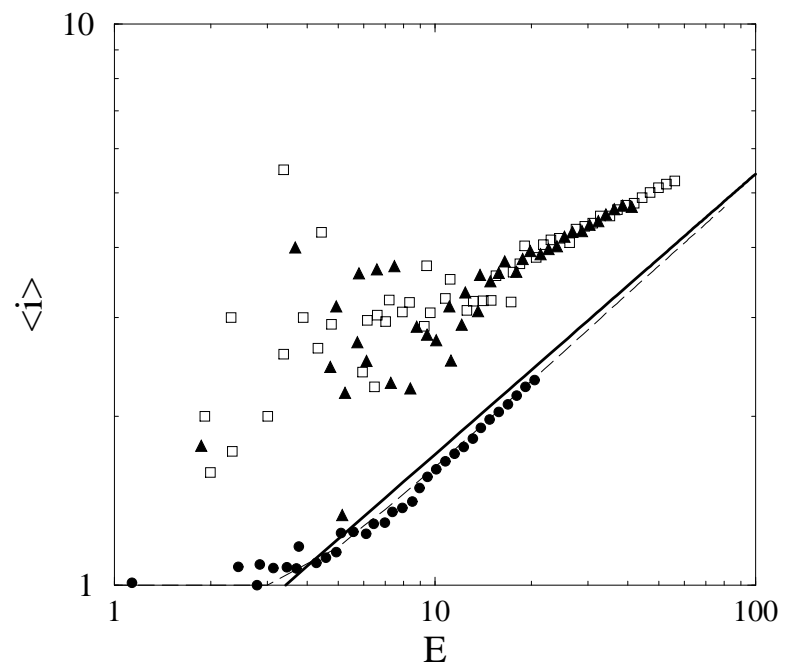

FIG. 2. Number of electron above the Fermi level as a function of energy. Solid dots correspond to a non-interacting system. Triangles correspond to a system of 465 sites and empty squares correspond to a system of 899 sites, both of them with Coulomb interactions. The dashed line is given by the discrete model and the solid line by the continuous one.

\section{EFFECTIVE NUMBER OF ELECTRONS PARTICIPATING IN LOW-ENERGY TRANSITIONS}

Let us consider that our system is in any of the $N(E)$ configurations up to energy $E$ with equal probability and let us study how the site occupancy depends on the interactions present. For the non-interacting system all configuration are related to at least another lower energy 
configuration by a single electron jump. This is no longer true for interacting systems. We can generalize this idea through the concept of the Hamming distance between two configurations, which is defined as the number of sites with different occupation in both configurations. In the non-interacting case, for any given configuration there is always at least one lower energy configuration whose Hamming distance is equal to 2 . We note that in the previous section we calculated the Hamming distance to the ground state measured in the number of electron jumps needed to reach this state from a given configuration. Now we try to estimate for interacting systems the (average) minimum Hamming distance from a given configuration to any lower energy configuration. Obviously this quantity will strongly affect the structure of phase space and in particular will influence the number of sites whose occupation changes in the $N(E)$ configurations.

For non-interacting systems following a Fermi-Dirac statistic, the importance of the number of sites whose occupation changes is reflected in the fact that, in the thermodynamic limit, the entropy $S=\ln N(E)$ can be expressed as a sum over the sites:

$$
S_{N}=-\sum_{i}^{n}\left(\left\langle s_{i}\right\rangle \ln \left\langle s_{i}\right\rangle+\left\langle 1-s_{i}\right\rangle \ln \left\langle 1-s_{i}\right\rangle\right),
$$

where $\left\langle s_{i}\right\rangle$ is the average occupation number at site $i$ and $n$ is the number of sites in the sample (note that sites with either $\left\langle s_{i}\right\rangle=0$ or 1 do not contribute to $S_{N}$ ). In general, for an interacting system, $S_{N}$ does not correspond to the real entropy. A very simple interacting case where it is possible to obtain a similar relation is a system whose elementary excitations consist of simultaneous jumps of $q$ electrons. If these excitations are independent, the number of sites contributing to the sum in Eq. (23) is $q$ times the number for the non-interacting case. Thus, $S_{N}=q \ln N(E)$ is proportional to the real entropy. We could propose $S_{N} / \ln N(E)$ as a rough quantity to estimate $q$ but it is affected by the finite size effects observed in Fig. 1. To avoid this finite size effects, we propose the quantity $S_{N} / S_{N}^{(\mathrm{NI})}$, where $S_{N}^{(\mathrm{NI})}$ is $S_{N}$ for a non-interacting system, as a measure of the number of electrons participating in a low-energy transition. In Fig. 3, we plot $S_{N} / S_{N}^{(\mathrm{NI})}$ as a function of $\ln (N(E))$ for a system with short-range interactions of 899 sites (solids triangles), and for three sizes of a coulombic system: 899 sites (empty dots), 465 sites (diamonds) and 248 sites (empty squares). $S_{N}$ depends on the size of the system when interactions are present. For systems with short range interactions this dependence is much weaker and we only plot one size in Fig. 3 for the sake of clarity. These results nicely show the importance of correlations in the low-energy configurations of interacting systems.

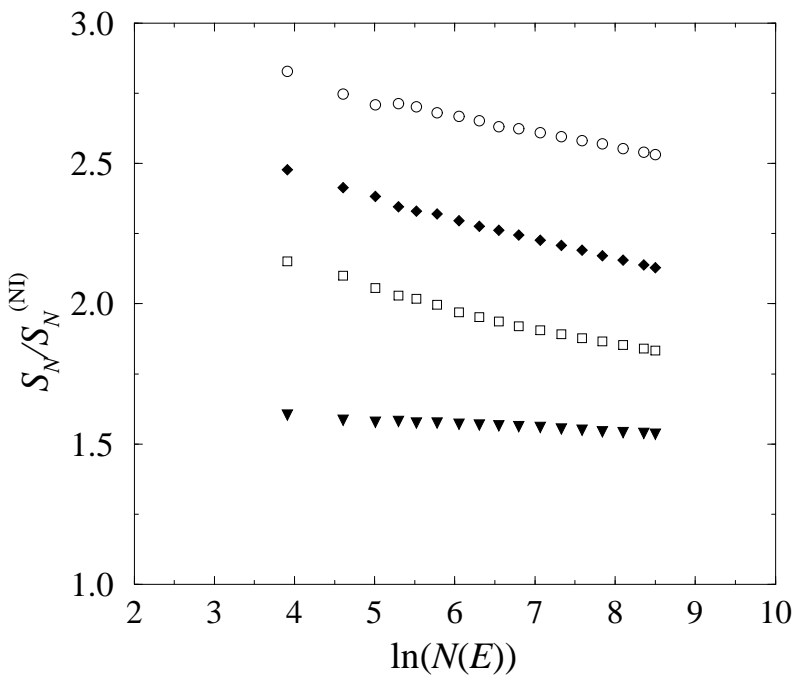

FIG. 3. $S_{N} / S_{N}^{(\mathrm{NI})}$ as a function of $\ln (N(E))$, where $N(E)$ is the number of low-energy configurations considered for systems with short range interactions of 899 sites (solids triangles) and systems with Coulomb interactions of 899 sites (empty dots), 465 sites (diamonds) and 248 sites (empty squares).

\section{VALLEYS IN CONFIGURATION SPACE}

To finalize, we study some aspects of the valley structure of the space of low-energy configurations for systems with Coulomb interactions. In this space we can define a separation (which does not have the metric properties of a distance) between configurations $I$ and $J$, with energies $E_{I}$ and $E_{J}$, respectively, through their transition rate $\omega_{I J}$, taken to be equal to [1]:

$$
\omega_{I J}=\frac{1}{\tau_{0}} \exp \left\{-2 \sum r_{i j} / a\right\} \exp \left\{\frac{E_{J}-E_{I}}{k T}\right\},
$$

for $E_{J}>E_{I}$, and without the second exponential for the opposite case. In Eq. (24), $\tau_{0}$ is the inverse phonon frequency, of the order of $10^{-13} \mathrm{~s}, a$ is the localization radius, and $\sum r_{i j}$ is the minimized sum of the hopping lengths of the electrons participating in the transition. 

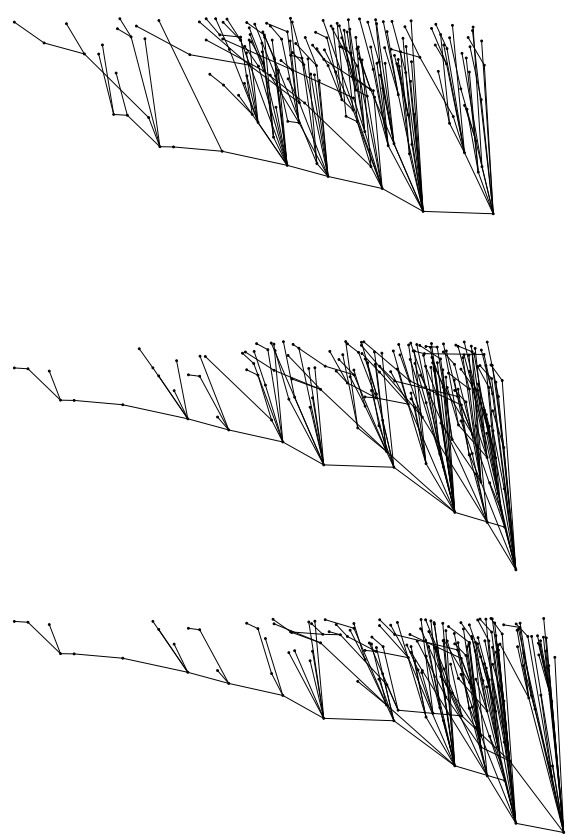

FIG. 4. Relaxation-trees in configuration space. Each dot represents a configuration and each line the fastest transition from the upper configuration, vertical distances represent their energies, being their mutual horizontal distance the logarithm of the transition time. The bottom of the valley is a different configuration for each case represented. The configurations considered as bottom of the valley are the ground state (figure at the bottom), the second configuration and the eighteenth configuration (figure at the top).

A way to study the valley structure is through the relaxation processes. More specifically, we assume a very small temperature so that from a given excited configuration we always go downwards in energy and we consider only the fastest transition rate at each step. Thus, we associate each configuration with a lower energy one and we construct in this way relaxation-trees. In Fig. 4 we show three different relaxation-trees in configuration space for the same sample. All transition jumps considered are plotted as lines where the horizontal distance is proportional to the logarithm of the transition time and the vertical one to the jump in energy. The bottom valley of the three cases represented are (in ascending order) the ground state, the second and the eighteenth configuration. At a qualitative level at least we can appreciate certain self-similarity between the trees.

Each metastable configuration, whose fastest transition downward in energy is carried out by the simultaneous jump of two or more electrons, corresponds to the bottom of a valley. It can be characterized by the number of electrons, $i$, participating in the fastest transition downward in energy. Its corresponding valley is the set of configurations that relax to it. For each characteristic number, $i$, let us define the integrated density of valleys, $N_{i}(E)$, as the number of $i$-valleys up to an energy $E$. The standard integrated density of configurations is, ob- viously, $N_{0}(E)=\sum_{i=1} N_{i}(E)$, where in order to use a closed notation we denote by $N_{0}$ to the standard integrated density of configurations and by $N_{1}$ to the integrated density of configurations whose fastest transition is a one electron jump. We have numerically checked that these integrated densities fit fairly well an expression of the form

$$
N_{i}(E)=C \exp \left\{\left(E / E_{0}\right)^{\alpha}\right\}
$$

where $C$ and $E_{0}$ depend on the characteristic number of electrons. On the other hand, the exponent $\alpha$ does not seem to depend on the characteristic number of electrons although it presents very large fluctuations from sample to sample. For the range of energies studied this exponent is roughly 0.7 , the exponent for the integrated density of configurations $N_{0}(E)$ at the energy interval considered. We know from a previous section that $\alpha=0.5$ in the thermodynamic limit. The proportion of metastable states with respect to normal configurations decreases with increasing energy. In Fig. 5, the density of configurations $N_{0}(E)$ (empty squares) and the density of valleys stables against 2 (solid dots), 3 (solid diamonds) and 4 (solid triangles) simultaneous transitions are plotted as a function of energy, $E$. All curves fit fairly well the expression $N(E)=C \exp \left(E / E_{0}\right)^{\alpha}$, with different $C$ and $E_{0}$ but with an exponent $\alpha$ very close to 0.7 in all cases.

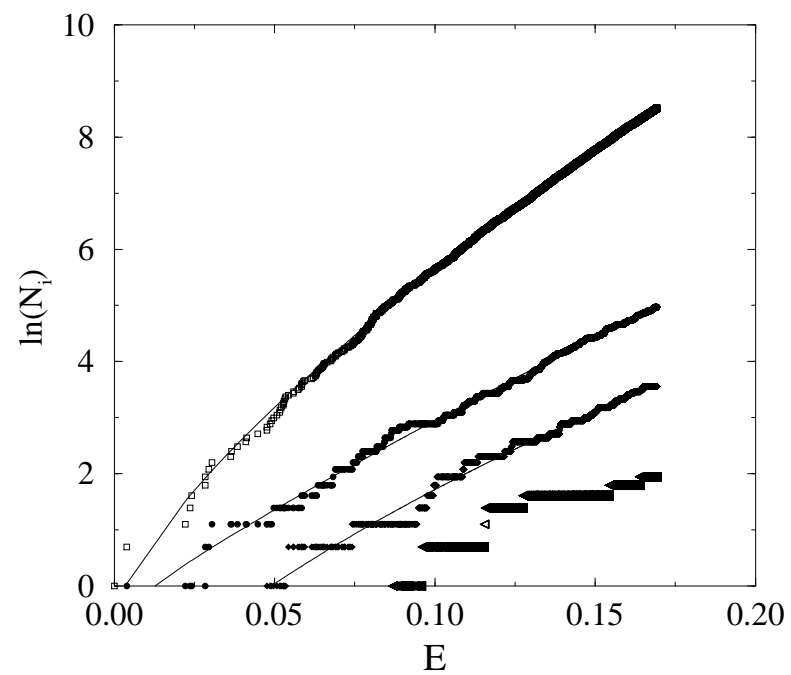

FIG. 5. Integrated densities of configurations $N_{i}(E)$ versus energy $E$. Empty squares corresponds to the integrated density of configurations, $i=0$, while the other curves correspond to integrated densities of metastable configurations with different characteristic number of electrons: 2 (solid dots), 3 (solid diamonds) and 4 (empty triangles). All curves fit fairly well the expression $N(E)=C \exp \left(E / E_{0}\right)^{\alpha}$, with different $C$ and $E_{0}$ but with an exponent $\alpha$ close to 0.7 in all cases. 


\section{CONCLUSIONS}

We have calculated the integrated density of configurations for systems with no interactions, short range interactions and Coulomb interactions. A comparation of our numerical results with a continuous theoretical model shows important finite size effects. However, our data overlap fairly well with a discrete model in the noninteracting case. The finite size effects found in the noninteracting case could serve to estimate these effects in interacting systems. We have more deeply studied the structure of phase space analyzing other quantities like $\langle i\rangle$ (half the average Hamming distance from a given configuration to the ground state), $S_{N}$ (the "site occupation entropy" see Eq. (23)) and the valleys in configuration space. For a given energy, $\langle i\rangle$ strongly depends on the type of interactions while, for the systems considered, it does not depend on the system size. The "site occupation entropy", $S_{N}$, increases with the strength of interactions and, for a long range one, with the size of the system.

\section{ACKNOWLEDGMENTS}

We thank Prof. M. Pollak for useful discussions. We would like to acknowledge financial support from the Spanish DGES, project numbers PB96-1118 and PB961120, and from the Fundación Séneca, Región de Murcia. A.P.G acknowledges CajaMurcia for a grant.

[1] M. Pollak and M. Ortuño, in Electron-Electron Interactions in Disordered Systems, edited by A.L. Efros and M. Pollak (North-Holland, Amsterdam, 1985).

[2] S. D. Baranovskii, A. L. Efros, B. L. Gelmont and B. I. Shklovskii, J. Phys. C, 121023 (1979).

[3] J. H. Davies, P. A. Lee and T. M. Rice, Phys. Rev. B, 294260 (1984).

[4] B.I. Shklovskii and A.L. Efros, Electronic Properties of Doped Semiconductors (Springer, Heidelberg, 1984).

[5] W. Mason, S. V. Kravchenko, G. E. Bowker and J. E. Furneaux, Phys. Rev. B, 527857 (1995)

[6] C. J. Adkins, J. Phys. C, 11253 (1989).

[7] G. Pignatel and S. Sanguinetti, J. Phys. C, 5191 (1993).

[8] A. Pérez-Garrido, M. Ortuño, E. Cuevas, J. Ruiz and M. Pollak, Phys. Rev. B, 55 R8630 (1997).

[9] M. Mochena and M. Pollak, Phys. Rev. Lett., 67109 (1991).

[10] M. Schreiber and K. Tenelsen, Europhys. Lett., 21697 (1993).

[11] M. Ortuño, E. Cuevas, J. Ruiz and A. Pérez-Garrido, in Proceedings of the 6th international conference on Hop- ping and Related Phenomena, edited by O. Millo and Z. Ovadyahu, p. 35, (Racah Institute, Jerusalem 1995).

[12] K. Huang, in Statistical Mechanics Wiley, (1963) 